\title{
Preparation of an Exfoliated Graphite by Mechano-Chemical Processing, in the Presence of Sulfur
}

\author{
G Tarango-Rivero ${ }^{1}$, CD Gómez-Esparza ${ }^{1}$, D Lardizabal-Gutierrez ${ }^{1}$, C Carreño-Gallardo ${ }^{1}$, I Estrada- \\ Guel $^{1 *}$ and R Martínez-Sánchez ${ }^{1}$. \\ 1. Centro de Investigación en Materiales Avanzados (CIMAV). Laboratorio Nacional de Nanotecnología \\ Miguel de Cervantes, Chihuahua, Chih., Mexico. \\ * Corresponding author: ivanovich.estrada@cimav.edu.mx
}

Graphite (Gr) is one of the most chemically inert materials which resist most alkalis, acids and corrosive gases, but its reactivity increases with the temperature reacting with oxygen at temperatures above 400 $1200^{\circ} \mathrm{C}[1]$, due among other things to its peculiar hexagonal array and a layered, planar structure where the individual layers are named graphenes. Isolated graphene has attracted extensive attention for practical applications due to their excellent electrical, mechanical and chemical properties [2]. Taking advantage of the relative weakness of Van Der Walls interactions, it is possible to obtain graphenes from graphite in form of exfoliated graphite (EG). On the other hand, sulfur (S) is an abundant nonmetallic element and an essential component of all living cells, it is known since ancient times. At the present, it is used as a sustainable and cheap alternative to replenish S lots from soils, avoiding soil and water pollution [3].

The present study deals with a route based on a solid-phase exfoliation of natural graphite to produce EGs using a mechano-chemical procedure based on high-energy ball milling. As raw material we use natural Gr flakes and pure S as exfoliating reagents. Some Gr-S equimolar mixtures were milled in a SPEX device for $8 \mathrm{~h}$. Then, $\mathrm{S}$ was separated from the milled powders in order to obtain a porous highly defoliated material using thermal processing. The milled samples were heated in a tube furnace at $350^{\circ} \mathrm{C}$ during $2 \mathrm{~h}$ under a protective inert Ar atmosphere. Under these conditions, S sublimates and condenses on the cool ends of the tube. After the system reach room temperature, the mixtures were separated and characterized.

In the thermogram of Fig. 1a, is evident a different thermal behavior of components at $350^{\circ} \mathrm{C}$, while pure sulfur loses $99.1 \%$ of its mass (red curve), pure graphite practically does not lose mass during the heating process (only $0.5 \%$, blue curve), based on this behavior is possible to separate both components using a relatively simple heating process (solvent free). In fact, the Gr-S mixture (black curve) suffers a mass loss of $68.8 \%$, which indicates that almost all the sulfur has been removed (near $95 \%$ efficiency). In the adsorption-desorption curves (1b), is noticeable a huge increase of surface area (SA) of EG using this mechanochemical process $\left(170 \mathrm{~m}^{2} / \mathrm{g}\right)$ compared with a single mechanical route $\left(12 \mathrm{~m}^{2} / \mathrm{g}\right)$ run at same time. Fig. 2 shows SEM micrographs and EDS mappings of the Gr-S samples before and after the heating process, in the SEM images (2a and $2 b$ ) we can notice important changes on the morphology of the samples and presence of isolated graphite layers after heating. EDS mappings shows a homogeneous distribution of S and Gr (2c) in the milled samples, after heating S is removed, leaving an almost pure Gr residue $(2 \mathrm{~d})$.

References:

[1] A Theodosiou, AN Jones and BJ Marsden, PLOS ONE 12(8) (2017), p. 1.

[2] AK Geim and KS Novoselov, Nature Materials 6 (2007), p. 183.

[3] A Reis Lucheta and M Rodrigues Lambais, Rev. Bras. Ciênc. Solo 36(5) (2012), p.1369 

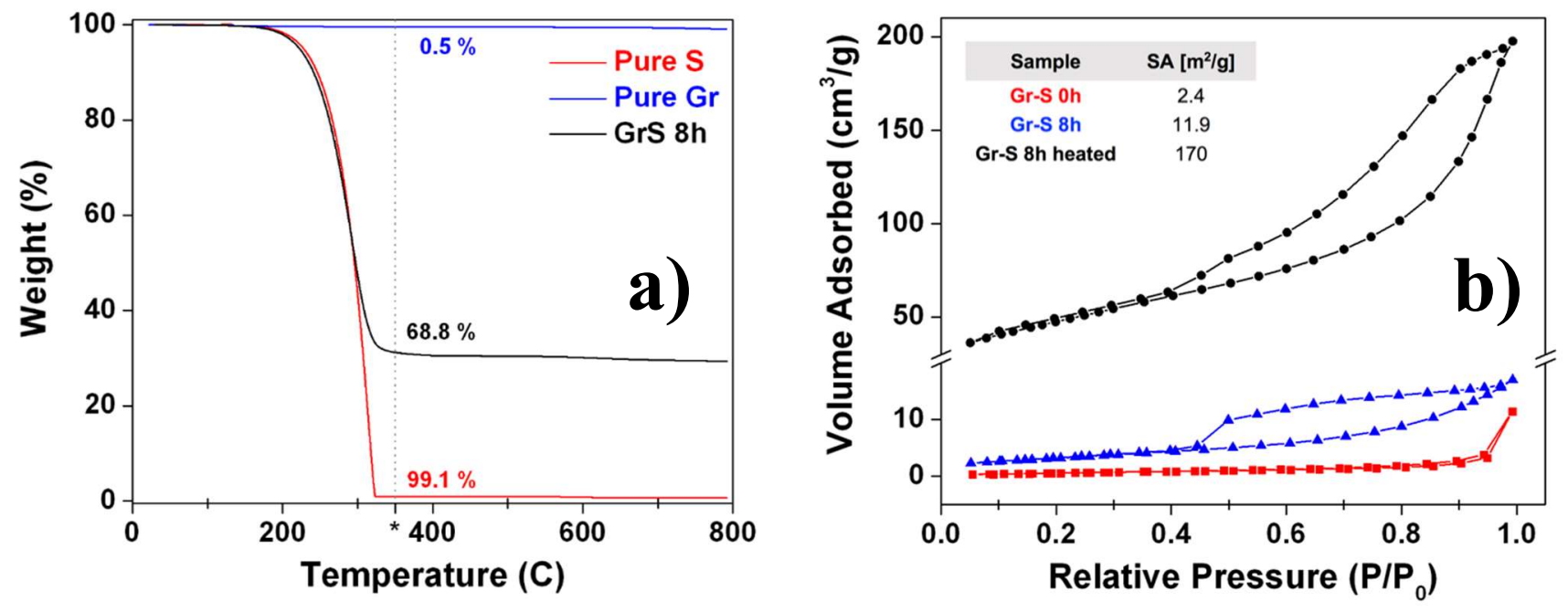

Figure 1. Thermogram (a) and adsorption-desorption isotherms (b) with summary chart of un milled $(0 \mathrm{~h})$, milled (8h) and milled-heated samples.
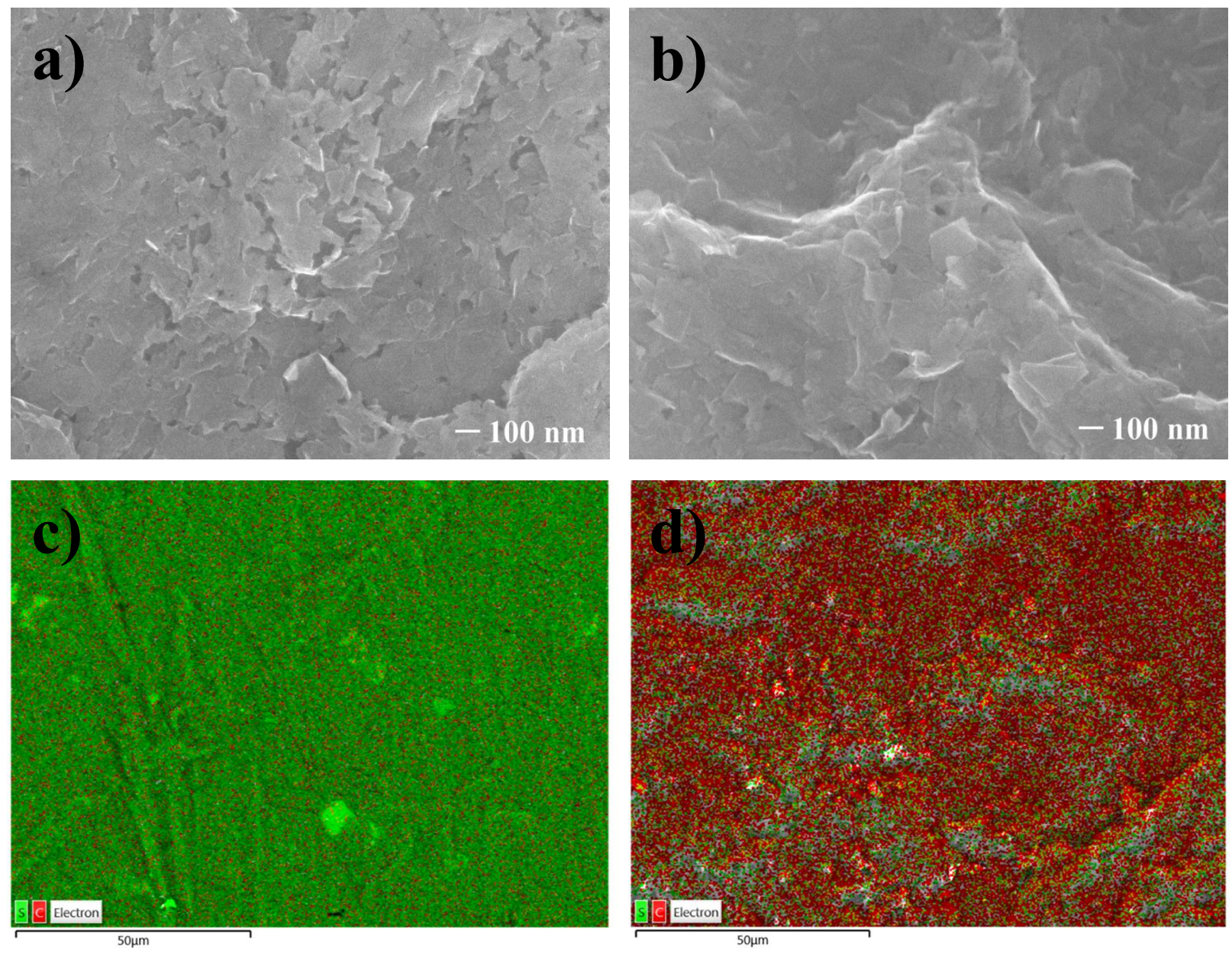

Figure 2. SEM micrographs and low magnification elemental maps of Gr-S samples: milled (a and c) and heated (b and d). In the bottom EDS mappings, sulfur is indicated in green and carbon in red color. 